\title{
MULTILINEAR EXPONENTIAL SUMS WITH A GENERAL CLASS OF WEIGHTS
}

\author{
BRYCE KERR AND SIMON MACOURT
}

\begin{abstract}
In this paper we obtain some new estimates for multilinear exponential sums in prime fields with a more general class of weights than previously considered. Our techniques are based on some recent progress of Shkredov in Additive Combinatorics with roots in Rudnev's point plane incidence bound. We apply our estimates to obtain new results concerning exponential sums with sparse polynomials and Weyl sums over small generalized arithmetic progressions.
\end{abstract}

\section{INTRODUCTION}

Given a prime number $p$, subsets $\mathcal{X}_{1}, \ldots, \mathcal{X}_{n} \subseteq \mathbb{F}_{p}^{*}$ and sequences of complex numbers $\omega_{1}(\mathbf{x}), \ldots, \omega_{n}(\mathbf{x})$, we define the weighted multilinear exponential sum over $n$ variables by

$$
S\left(\mathcal{X}_{1}, \ldots, \mathcal{X}_{n} ; \omega_{1}, \ldots, \omega_{n}\right)=\sum_{x_{1} \in \mathcal{X}_{1}} \ldots \sum_{x_{n} \in \mathcal{X}_{n}} \omega_{1}(\mathbf{x}) \ldots \omega_{n}(\mathbf{x}) \mathbf{e}_{p}\left(x_{1} \ldots x_{n}\right),
$$

where $\omega_{i}$ are $n-1$ dimensional weights that depend on all but the $i$ th variable and $\mathbf{e}_{p}(u)=\exp (2 \pi i u / p)$. Assuming each $\left|\omega_{i}(\mathbf{x})\right| \leqslant 1$, we are interested in obtaining upper bounds of the form

$$
\left|S\left(\mathcal{X}_{1}, \ldots, \mathcal{X}_{n} ; \omega_{1}, \ldots, \omega_{n}\right)\right| \leqslant X_{1} \ldots X_{n} p^{-\delta},
$$

where $\left|\mathcal{X}_{i}\right|=X_{i}$. The first result in this direction is Vinogradov's bilinear estimate and states that

$$
\left|\sum_{x_{1} \in X_{1}} \sum_{x_{2} \in X_{2}} \omega_{1}\left(x_{2}\right) \omega_{2}\left(x_{1}\right) e_{p}\left(x_{1} x_{2}\right)\right| \leqslant p^{1 / 2} X_{1}^{1 / 2} X_{2}^{1 / 2},
$$

which is nontrivial provided $X_{1} X_{2}>p$. For values of $n \geqslant 3$ progress has been made through Additive Combinatorics with the first results due to Bourgain, Glibichuck and Konyagin [7] under some restrictions on the sets, weights and number of variables occuring in (1.1) although

2010 Mathematics Subject Classification. 11L07, 11T23.

Key words and phrases. exponential sum, sparse polynomial. 
their result was general enough to obtain new estimates for sums over small subgroups. Bourgain [3] extended the results of [7] and obtained an optimal result with respect to the size of $X_{1} \ldots X_{n}$. In particular, Bourgain showed that for all $\varepsilon>0$ there exists a $\delta>0$ such that

$$
\sum_{x_{1} \in \mathcal{X}_{1}} \ldots \sum_{x_{n} \in \mathcal{X}_{n}} e_{p}\left(x_{1} \ldots x_{n}\right) \ll X_{1} \ldots X_{n} p^{-\delta}
$$

provided

$$
X_{i}>p^{\varepsilon}, \quad X_{1} \ldots X_{n} \geqslant p^{1+\varepsilon}
$$

and we note that Bourgain gives the dependence of $\delta$ on $\varepsilon$. Recently, Shkredov [26] has made significant quantitative improvements to the results of Bourgain by exploiting a direct connection with geometric incidence estimates of Rudnev [23]. Of particular relevance are the results of Petridis and Shparlinski [22] and Macourt [18] for recent estimates of three and four dimensional multilinear sums and Shkredov [25] for the sharpest current results for exponential sums over subgroups of medium size. We mention that a direct application of the methods from $[18,22]$ are unable to give bounds for multilinear sums beyond four dimensional sums. However, in this paper we are able to break through this barrier and apply related techniques to given new nontrivial results for multilinear sums beyond four variables.

Given a set $\mathcal{A} \subseteq \mathbb{F}_{p}$ and an integer $k$ we let $D_{k}^{\times}(\mathcal{A})$ count the number of solutions to the equation

$\left(a_{1}-a_{2}\right)\left(a_{3}-a_{4}\right) \ldots\left(a_{2 k-1}-a_{2 k}\right)=\left(b_{1}-b_{2}\right)\left(b_{3}-b_{4}\right) \ldots\left(b_{2 k-1}-b_{2 k}\right)$,

for $a_{i}, b_{i} \in \mathcal{A}$. The quantity $D_{k}(\mathcal{A})$ plays an important role in our arguments and we obtain some new estimates for $D_{k}(\mathcal{A})$, one of which improves the error term in a result of Shkredov [26, Theorem 32] for sets of cardinality $|\mathcal{A}| \geqslant p^{1 / 2}$. We then apply our estimates to obtain some new bounds for sums of the form (1.1) which are motivated by applications to exponential sums with sparse polynomials and Weyl sums over small generalized arithmetic progressions.

Given tuples of integers $a_{1}, \ldots, a_{t}$ and $k_{1}, \ldots, k_{t}$ we define the $t$ sparse polynomial

$$
\Psi(X)=\sum_{i=1}^{t} a_{i} X^{k_{i}}
$$


and consider the exponential sums

$$
T_{\chi}(\Psi)=\sum_{x \in \mathbb{F}_{p}^{*}} \chi(x) \mathbf{e}_{p}(\Psi(x)) .
$$

Multinomial exponential sums of the form (1.4) have been studied extensively. We first note by the Weil bound, see [28, Appendix 5, Example 12]

$$
T_{\chi}(\Psi) \leqslant p^{1 / 2} \max \left(k_{1}, \ldots, k_{t}\right) .
$$

When $k_{1}, \ldots, k_{t}$ are small the above estimate is sharp and we consider the case when $\max \left(k_{1}, \ldots, k_{t}\right)$ grows with $p$. In this setting, progress on the simplest case of monomials was first made by Shparlinski [27] and was further improved by Heath-Brown and Konyagin [16] using techniques based on Stepanov's method, although the current sharpest estimates are based on Additive Combinatorics, see for example $[7,8]$. More general sums of the form (1.4) were first considered by Mordell [20] and are often referred to as Mordell's exponential sum and we refer the reader to [1,9-14] for previous estimates of these sums. We also mention the cases of trinomials and quadrinomials have been given new bounds in [17] and [19] and we follow these techniques to reduce to multilinear sums of the form (1.1).

A second application of our bound for the sums (1.1) is a new estimate Weyl sums over small generalized arithmetic progressions. Generalized arithmetic progressions are defined as sets of the form

$$
\mathcal{A}=\left\{\alpha_{1} h_{1}+\cdots+\alpha_{r} h_{r}+\beta: 1 \leqslant h_{i} \leqslant H_{i}\right\} .
$$

For $\mathcal{A}$ as above, we define the rank of $\mathcal{A}$ to be $r$ and say that $\mathcal{A}$ is proper if

$$
|\mathcal{A}|=H_{1} \ldots H_{r} .
$$

Shao [24] has previously shown that for any polynomial $F$ we have

$$
\sum_{a \in \mathcal{A}} \mathbf{e}_{p}(F(a)) \ll_{r} p^{1 / 2+o(1)}
$$

which can be considered a Pólya-Vinogradov type estimate for generalised arithmetic progressions. We use our estimates for (1.1) to obtain a power saving for Weyl sums over proper generalized arithmetic progressions with an essentially optimal range on the cardinality of $\mathcal{A}$, see Theorem 1.4.

For the entirety of this paper we let $\left|\mathcal{X}_{i}\right|=X_{i}$, and similarly for other sets $|\mathcal{Y}|=Y$. We also use the notation $A \ll B$ to indicate $A \leqslant c|B|$ 
for some absolute constant $c$ and similarly $A \ll_{k} B$ to mean the same where $c$ depends on some parameter $k$.

1.1. Main Results. In what follows we keep notation as in (1.1).

Theorem 1.1. Let $n \geqslant 4, \mathcal{X}_{i} \subset \mathbb{F}_{p}^{*}$ subsets satisfying

$$
\left|\mathcal{X}_{i}\right|=X_{i}, \quad X_{1} \geqslant X_{2} \geqslant \cdots \geqslant X_{n},
$$

and

$$
X_{1} X_{n}^{1 / 2} \leqslant p
$$

Then we have

$$
\begin{aligned}
& S\left(\mathcal{X}_{1}, \ldots, \mathcal{X}_{n} ; \omega_{1}, \ldots, \omega_{n}\right) \\
& \ll_{n} X_{1} \ldots X_{n}\left(\frac{1}{X_{1}^{1 / 2}}+\cdots+\frac{1}{X_{n}^{1 / 2^{n}}}+p^{\frac{1}{2^{n}}} X_{1}^{-\frac{1}{2^{n}}} X_{n}^{-\frac{1}{2^{n+1}}} \prod_{i=2}^{n-1} B_{n}\left(\mathcal{X}_{i}\right)\right)
\end{aligned}
$$

where

$$
B_{n}(\mathcal{X})= \begin{cases}p^{\frac{1}{2^{2 n-3}(n-2)}} X^{-\frac{2^{n-2}+1}{2^{2 n-3}(n-2)}+o(1)}, & \text { if } p^{\frac{1}{2}+\frac{1}{2^{n-1}+2}} \geqslant X \geqslant p^{\frac{217}{433}}, \\ X^{-\frac{2^{n-2}-1+2 c_{1}}{2^{2 n-3}(n-2)}+o(1)}, & \text { if } p^{\frac{217}{433}}>X \geqslant p^{\frac{48}{97}}, \\ X^{-\frac{2^{n-2}-1+2 c_{2}}{2^{2 n-3}(n-2)}+o(1)}, & \text { if } X<p^{\frac{48}{97}}\end{cases}
$$

and $c_{1}=\frac{1}{434}$ and $c_{2}=\frac{1}{192}$.

We give an example of when Theorem 1.1 is nontrivial. Suppose $n=6$ and $X_{1}=X_{2}=\cdots=X_{6} \leqslant p^{\frac{48}{97}}$. Then we have

$$
S\left(\mathcal{X}_{1}, \ldots, \mathcal{X}_{6} ; \omega_{1}, \ldots, \omega_{6}\right) \ll p^{\frac{1}{64}} X_{1}^{\frac{3110399}{524288}+o(1)} .
$$

One can see that this is stronger than the trivial bound

$$
S\left(\mathcal{X}_{1}, \ldots, \mathcal{X}_{6} ; \omega_{1}, \ldots, \omega_{6}\right) \ll X_{1}^{6}
$$

for $X_{1}>p^{8 / 27}$. In the case of sets of cardinality a little larger than $p^{1 / 2}$ we can obtain sharper estimates.

Theorem 1.2. Let $\mathcal{X}_{i} \subset \mathbb{F}_{p}$ satisfy $\left|\mathcal{X}_{i}\right|=X_{i}, X_{1} \geqslant X_{2} \geqslant \cdots \geqslant X_{n}$

$$
\left|\mathcal{X}_{i}\right| \geqslant p^{1 / 2+1 /\left(2^{n+1}-6\right)} \text {. }
$$

Then we have

$$
\begin{aligned}
& \left|S\left(\mathcal{X}_{1}, \ldots, \mathcal{X}_{n} ; \omega_{1}, \ldots, \omega_{n}\right)\right| \ll_{n} \\
& X_{1} \ldots X_{n}\left(\frac{1}{X_{1}^{1 / 2}}+\cdots+\frac{1}{X_{n}^{1 / 2^{n}}}+p^{o(1)}\left(\frac{p^{1 / 2}}{\left(X_{1} \ldots X_{n}\right)^{1 / n}}\right)^{1 / 2^{n}}\right)
\end{aligned}
$$


The following is a consequence of Theorem 1.1 .

Theorem 1.3. Let $\Psi(X)$ be a multinomial of the form (1.3), with coefficients $a_{i} \in \mathbb{F}_{p}^{*}$ for $i=1, \ldots, t$. We define

$$
\alpha_{k_{i}}=\operatorname{gcd}\left(k_{i}, p-1\right)
$$

and

$$
\beta_{k_{i}}=\frac{\alpha_{k_{i}}}{\operatorname{gcd}\left(\alpha_{k_{i}}, \alpha_{k_{t}}\right)}
$$

Suppose $\beta_{k_{1}} \geqslant \cdots \geqslant \beta_{k_{t-1}}$. Then

$$
\begin{aligned}
& T_{\chi}(\Psi) \\
& \ll p\left(\left(\frac{\alpha_{k_{t}}}{p-1}\right)^{\frac{1}{2}}+\beta_{k_{1}}^{\frac{-1}{2^{2}}}+\cdots+\beta_{k_{t-1}}^{\frac{-1}{2^{t}}}+p^{\frac{1}{2^{t}}} C_{t}\left(\alpha_{k_{t}}\right) \prod_{i=1}^{t-2} D_{t}\left(\beta_{k_{i}}\right)\right)
\end{aligned}
$$

where

$$
C_{t}(\alpha)= \begin{cases}\alpha^{\frac{3}{2^{t+1}}} p^{-\frac{3}{2^{t+1}}}, & \text { if } \alpha \geqslant p^{\frac{1}{2}} \log p \\ \alpha^{\frac{1}{2^{t+1}}} p^{-\frac{1}{2^{t}}}, & \text { if } \alpha<p^{\frac{1}{2}} \log p\end{cases}
$$

and

$$
D_{t}(\beta)= \begin{cases}p^{-\frac{1}{2^{t}(t-2)}}, & \text { if } \beta \geqslant p^{\frac{1}{2}} \log p, \\ \beta^{-\frac{1}{2^{t-1}(t-2)}}, & \text { if } \beta<p^{\frac{1}{2}} \log p .\end{cases}
$$

We mention that Theorem 1.3 returns the same bound as [17, Theorem 1.1] when $t=4$. We also mention the strength in this bound is that it relies on mutual greatest common divisors, rather than the size of the exponents. With this in mind, one can give examples of when this is stronger than all known bounds for a given $t$ by first ensuring that $\alpha_{k_{t}}$ is small and each of the powers are large. We direct the reader to [17, Corollary 1.2] for such an example for the case $t=4$.

Combining ideas from the proof of Theorem 1.1 with estimates of Bourgain for multilinear sums we extend a result of Shao [24] to the setting of Weyl sums over small generalized arithmetic progressions.

Theorem 1.4. Let $p$ be prime, $\mathcal{A} \subseteq \mathbb{F}_{p}$ a proper generalized arithmetic progression of rank $r$ and $F \in \mathbb{F}_{p}[X]$ a polynomial of degree $d$. For any $\varepsilon>0$ there exists some $\delta>0$ such that if

$$
|\mathcal{A}| \geqslant p^{1 / d+\varepsilon}
$$

then

$$
\sum_{a \in \mathcal{A}} e_{p}(F(a)) \ll_{r, d}|\mathcal{A}| p^{-\delta}
$$


We note the condition $|\mathcal{A}| \geqslant p^{1 / d+\varepsilon}$ is sharp which may be seen by considering the example

$$
\mathcal{A}=\left\{1,2, \ldots,\left\lfloor p^{1 / d} / 10\right\rfloor\right\}, \quad F(x)=x^{d},
$$

so that

$$
\sum_{a \in \mathcal{A}} e_{p}(F(a)) \gg|\mathcal{A}|
$$

\section{Multilinear Exponential Sums}

2.1. Reduction mean values. The following result is a variant of [22, Lemma 2.10] which is more suitable for applications to exponential sums when the variables may run through sets of differing cardinalities.

Lemma 2.1. Let $n \geqslant 2$. Suppose $S\left(\mathcal{X}_{1}, \ldots, \mathcal{X}_{n} ; \omega_{1}, \ldots, \omega_{n}\right)$ is defined as in (1.1). Then

$$
\begin{gathered}
\left|S\left(\mathcal{X}_{1}, \ldots, \mathcal{X}_{n} ; \omega_{1}, \ldots, \omega_{n}\right)\right|^{2^{n-1}} \ll\left(X_{1} \ldots X_{n}\right)^{2^{n-1}}\left(\frac{1}{X_{n}^{2^{n-2}}}+\cdots+\frac{1}{X_{2}}\right) \\
+X_{1}^{2^{n-1}-1}\left(X_{2} \ldots X_{n}\right)^{2^{n-1}-2} \sum_{\substack{x_{2}, y_{2} \in \mathcal{X}_{2} \\
x_{2} \neq y_{2}}} \ldots \sum_{\substack{x_{n}, y_{n} \in \mathcal{X}_{n} \\
x_{n} \neq y_{n}}} \\
\times\left|\sum_{x_{1} \in \mathcal{X}_{1}} \mathbf{e}_{p}\left(x_{1}\left(x_{2}-y_{2}\right) \ldots\left(x_{n}-y_{n}\right)\right)\right| .
\end{gathered}
$$

Proof. We proceed by induction on $n$ and first consider the case $n=2$. Our sums take the form

$$
S\left(\mathcal{X}_{1}, \mathcal{X}_{2}, \omega_{1}, \omega_{2}\right)=\sum_{x_{1} \in \mathcal{X}_{1}} \sum_{x_{2} \in \mathcal{X}_{2}} \omega_{1}\left(x_{2}\right) \omega_{2}\left(x_{1}\right) e_{p}\left(x_{1} x_{2}\right),
$$

and hence by the Cauchy-Schwarz inequality

$$
\left|S\left(\mathcal{X}_{1}, \mathcal{X}_{2}, \omega_{1}, \omega_{2}\right)\right|^{2} \leqslant X_{1} \sum_{x_{1} \in \mathcal{X}_{1}}\left|\sum_{x_{2} \in \mathcal{X}_{2}} e_{p}\left(x_{1} x_{2}\right)\right|^{2}
$$

Expanding the square, interchanging summation and isolating the diagonal contribution, we get

$$
\left|S\left(\mathcal{X}_{1}, \mathcal{X}_{2}, \omega_{1}, \omega_{2}\right)\right|^{2} \leqslant X_{1}^{2} X_{2}+X_{1} \sum_{\substack{x_{2}, y_{2} \in \mathcal{X}_{2} \\ x_{2} \neq y_{2}}}\left|\sum_{x_{1} \in \mathcal{X}_{1}} e_{p}\left(x_{1}\left(x_{2}-y_{2}\right)\right)\right|
$$


Suppose the statement of Lemma 2.1 is true for some integer $n-1 \geqslant$ 2 and consider the sums $S\left(\mathcal{X}_{1}, \ldots, \mathcal{X}_{n} ; \omega_{1}, \ldots, \omega_{n}\right)$. By the CauchySchwarz inequality

$$
\begin{aligned}
& \left|S\left(\mathcal{X}_{1}, \ldots, \mathcal{X}_{n} ; \omega_{1}, \ldots, \omega_{n}\right)\right|^{2} \leqslant X_{1} \ldots X_{n-1} \\
& \sum_{\substack{x_{i} \in \mathcal{X}_{i} \\
1 \leqslant i \leqslant n-1}}\left|\sum_{x_{n} \in \mathcal{X}_{n}} \omega_{1}(\mathbf{x}) \ldots \omega_{n-1}(\mathbf{x}) e_{p}\left(x_{1} \ldots x_{n}\right)\right|^{2}
\end{aligned}
$$

which after expanding the square, interchanging summation and isolating the diagonal contribution results in

$$
\begin{aligned}
\left|S\left(\mathcal{X}_{1}, \ldots, \mathcal{X}_{n} ; \omega_{1}, \ldots, \omega_{n}\right)\right|^{2} & \leqslant \frac{\left(X_{1} \ldots X_{n}\right)^{2}}{X_{n}} \\
& +X_{1} \ldots X_{n-1} \sum_{\substack{x_{n}, y_{n} \in \mathcal{X}_{n} \\
x_{n} \neq y_{n}}} S\left(x_{n}, y_{n}\right),
\end{aligned}
$$

where

$$
\begin{aligned}
& S\left(x_{n}, y_{n}\right)= \\
& \left|\sum_{\substack{x_{i} \in \mathcal{X}_{i} \\
1 \leqslant i \leqslant n-1}} \omega_{1}^{\prime}\left(\mathbf{x}^{\prime}, x_{n}, y_{n}\right) \ldots \omega_{n-1}^{\prime}\left(\mathbf{x}^{\prime}, x_{n}, y_{n}\right) e_{p}\left(x_{1} \ldots x_{n-1}\left(x_{n}-y_{n}\right)\right)\right|,
\end{aligned}
$$

and

$$
\mathbf{x}^{\prime}=\left(x_{1}, \ldots, x_{n-1}\right), \quad \omega_{j}\left(\mathbf{x}^{\prime}, x_{n}, y_{n}\right)=\omega_{j}\left(\mathbf{x}^{\prime}, x_{n}\right) \bar{\omega}_{j}\left(\mathbf{x}^{\prime}, y_{n}\right) .
$$

By Hölder's inequality

$$
\begin{aligned}
&\left|S\left(\mathcal{X}_{1}, \ldots, \mathcal{X}_{n} ; \omega_{1}, \ldots, \omega_{n}\right)\right|^{2^{n-1}} \ll \frac{\left(X_{1} \ldots X_{n}\right)^{2^{n-1}}}{X_{n}^{2^{n-2}}} \\
&+\left(X_{1} \ldots X_{n-1}\right)^{2^{n-2}} X_{n}^{2^{n-1}-2} \sum_{\substack{x_{n}, y_{n} \in \mathcal{X}_{n} \\
x_{n} \neq y_{n}}} S\left(x_{n}, y_{n}\right)^{2^{n-2}}
\end{aligned}
$$


We next fix some pair $x_{n} \neq y_{n}$ and apply our induction hypothesis to the sum $S\left(x_{n}, y_{n}\right)$. This gives

$$
\begin{aligned}
& S\left(x_{n}, y_{n}\right)^{2^{n-2}} \leqslant\left(X_{1} \ldots X_{n-1}\right)^{2^{n-2}}\left(\frac{1}{X_{n-1}^{2^{n-3}}}+\cdots+\frac{1}{X_{2}}\right) \\
& +X_{1}^{2^{n-2}-1}\left(X_{2} \ldots X_{n-1}\right)^{2^{n-2}-2} \\
& \quad \sum_{\substack{x_{i}, y_{i} \in \mathcal{X}_{i} \\
x_{i} \neq y_{i} \\
2 \leqslant i \leqslant n-1}}\left|\sum_{x_{1} \in \mathcal{X}_{1}} e_{p}\left(x_{1}\left(x_{2}-y_{2}\right) \ldots\left(x_{n-1}-y_{n-1}\right)\left(x_{n}-y_{n}\right)\right)\right|,
\end{aligned}
$$

which combined with the above implies

$$
\begin{aligned}
& \left|S\left(\mathcal{X}_{1}, \ldots, \mathcal{X}_{n} ; \omega_{1}, \ldots, \omega_{n}\right)\right|^{2^{n-1}} \leqslant\left(X_{1} \ldots X_{n}\right)^{2^{n-1}}\left(\frac{1}{X_{n}^{2^{n-2}}}+\cdots+\frac{1}{X_{2}}\right) \\
& +X_{1}^{2^{n-1}-1}\left(X_{2} \ldots X_{n}\right)^{2^{n-1}-2} \\
& \times \sum_{\substack{x_{i}, y_{i} \in \mathcal{X}_{i} \\
x_{i} \neq y_{i} \\
2 \leqslant i \leqslant n-1}}\left|\sum_{x_{1} \in \mathcal{X}_{1}} e_{p}\left(x_{1}\left(x_{2}-y_{2}\right) \ldots\left(x_{n-1}-y_{n-1}\right)\left(x_{n}-y_{n}\right)\right)\right|,
\end{aligned}
$$

and completes the proof.

We mention that the above proof is independent of the sizes of the $X_{i}$, and as such the lemma is left without such restrictions.

For any set $\mathcal{A} \subset \mathbb{F}_{p}$ we define

$D_{k}^{\times}(\mathcal{A})$

$$
=\left|\left\{\left(a_{1}-a_{2}\right) \ldots\left(a_{2 k-1}-a_{2 k}\right)=\left(b_{1}-b_{2}\right) \ldots\left(b_{2 k-1}-b_{2 k}\right): a_{i}, b_{i} \in \mathcal{A}\right\}\right|,
$$

and extend the notation when variables run through different sets by defining $D_{k}^{\times}\left(\mathcal{X}_{1}, \ldots, \mathcal{X}_{k}\right)$ to be the number of solutions to

$$
\left(w_{1}-x_{1}\right) \ldots\left(w_{k}-x_{k}\right)=\left(y_{1}-z_{1}\right) \ldots\left(y_{k}-z_{k}\right),
$$

for $w_{i}, x_{i}, y_{i}, z_{i} \in \mathcal{X}_{i}$. Finally, we use the notation $D_{k}^{\times, *}$ for the above cases where we exclude the solutions when the equation is 0 and define

$$
\widetilde{D}_{k}^{\times, *}\left(\mathcal{X}_{1}, \ldots, \mathcal{X}_{k}\right)=D_{k}^{\times, *}\left(\mathcal{X}_{1}, \ldots, \mathcal{X}_{k}\right)-\frac{\left(\prod_{i=1}^{k} X_{i}\left(X_{i}-1\right)\right)^{2}}{p-1} .
$$

We note that $\widetilde{D}_{k}^{\times, *}$ is the error in approximation of $D_{k}^{\times, *}$ by the expected main term.

Lemma 2.2. Let $\mathcal{X}_{1}, \ldots, \mathcal{X}_{k} \subset \mathbb{F}_{p}$. Then

$$
D_{k}^{\times, *}\left(\mathcal{X}_{1}, \ldots, \mathcal{X}_{k}\right) \leqslant\left(D_{k}^{\times, *}\left(\mathcal{X}_{1}\right) \ldots D_{k}^{\times, *}\left(\mathcal{X}_{k}\right)\right)^{1 / k}
$$


Proof. We let $K=D_{k}^{\times, *}\left(\mathcal{X}_{1}, \ldots, \mathcal{X}_{k}\right)$ and express $K$ in terms of multiplicative characters

$$
\begin{aligned}
K= & \sum_{w_{1}, x_{1}, y_{1}, z_{1} \in \mathcal{X}_{1}} \ldots \sum_{w_{k}, x_{k}, y_{k}, z_{k} \in \mathcal{X}_{k}} \\
& \frac{1}{p-1} \sum_{\chi \in \Omega} \chi\left(w_{1}-x_{1}\right) \ldots\left(w_{k}-x_{k}\right) \bar{\chi}\left(y_{1}-z_{1}\right) \ldots\left(y_{k}-z_{k}\right)
\end{aligned}
$$

where $\Omega$ is the set of all distinct characters. Clearly,

$$
K=\frac{1}{p-1} \sum_{\chi \in \Omega}\left|\sum_{w_{1}, x_{1} \in \mathcal{X}_{1}} \chi\left(w_{1}-x_{1}\right)\right|^{2} \cdots\left|\sum_{w_{k}, x_{k} \in \mathcal{X}_{k}} \chi\left(w_{k}-x_{k}\right)\right|^{2} .
$$

Using Holder's inequality, we obtain

$$
\begin{aligned}
K^{k} & \leqslant \frac{1}{(p-1)^{k}} \sum_{\chi \in \Omega}\left|\sum_{w_{1}, x_{1} \in \mathcal{X}_{1}} \chi\left(w_{1}-x_{1}\right)\right|^{2 k} \ldots \sum_{\chi \in \Omega}\left|\sum_{w_{k}, x_{k} \in \mathcal{X}_{k}} \chi\left(w_{k}-x_{k}\right)\right|^{2 k} \\
& =D_{k}^{\times, *}\left(\mathcal{X}_{1}\right) \ldots D_{k}^{\times, *}\left(\mathcal{X}_{k}\right) .
\end{aligned}
$$

The proof of the following is similar to that of Lemma 2.2 with summation only over non-principal characters.

Lemma 2.3. Let $\mathcal{X}_{1}, \ldots, \mathcal{X}_{k} \subset \mathbb{F}_{p}$. Then

$$
\widetilde{D}_{k}^{\times, *}\left(\mathcal{X}_{1}, \ldots, \mathcal{X}_{k}\right) \leqslant\left(\widetilde{D}_{k}^{\times, *}\left(\mathcal{X}_{1}\right) \ldots \widetilde{D}_{k}^{\times, *}\left(\mathcal{X}_{k}\right)\right)^{1 / k} .
$$

Using Lemma 2.1, Lemma 2.2 and Lemma 2.3 we give two general results relating estimates for $S\left(\mathcal{X}_{1}, \ldots, \mathcal{X}_{n} ; \omega_{1}, \ldots, \omega_{n}\right)$ to the quantities $D_{k}^{\times}(\mathcal{A})$ and $\widetilde{D}_{k}^{\times}(\mathcal{A})$. We first recall the classic Vinogradov bilinear estimate, see [6, Equation 1.4] or [15, Lemma 4.1].

Lemma 2.4. For any sets $\mathcal{X}, \mathcal{Y} \subseteq \mathbb{F}_{p}$ and any $\alpha=\left(\alpha_{x}\right)_{x \in \mathcal{X}}, \beta=$ $\left(\beta_{y}\right)_{y \in \mathcal{Y}}$ with

$$
\sum_{x \in \mathcal{X}}\left|\alpha_{x}\right|^{2}=A \quad \text { and } \quad \sum_{y \in \mathcal{Y}}\left|\beta_{y}\right|^{2}=B
$$

we have

$$
\left|\sum_{x \in \mathcal{X}} \sum_{y \in \mathcal{Y}} \alpha_{x} \beta_{y} \mathbf{e}_{p}(x y)\right| \leqslant \sqrt{p A B}
$$

Lemma 2.5. Let $n \geqslant 2$. Suppose $S\left(\mathcal{X}_{1}, \ldots, \mathcal{X}_{n} ; \omega_{1}, \ldots, \omega_{n}\right)$ is defined as in (1.1) and that

$$
X_{1} \geqslant X_{2} \cdots \geqslant X_{n}
$$


Then

$$
\begin{aligned}
\mid S\left(\mathcal{X}_{1}, \ldots,\right. & \left.\mathcal{X}_{n} ; \omega_{1}, \ldots, \omega_{n}\right)\left.\right|^{2^{n}} \ll\left(X_{1} \ldots X_{n}\right)^{2^{n}}\left(\frac{1}{X_{1}^{2^{n-1}}}+\cdots+\frac{1}{X_{n-1}^{2}}\right) \\
& +p X_{n}^{2^{n}-1}\left(X_{1} \ldots X_{n-1}\right)^{2^{n}-4}\left(D_{n-1}^{\times, *}\left(\mathcal{X}_{1}\right) \ldots D_{n-1}^{\times, *}\left(\mathcal{X}_{n-1}\right)\right)^{1 /(n-1)}
\end{aligned}
$$

Proof. Writing

$$
S=\sum_{\substack{x_{1}, y_{1} \in \mathcal{X}_{1} \\ x_{1} \neq y_{1}}} \ldots \sum_{\substack{x_{n-1}, y_{n-1} \in \mathcal{X}_{n-1} \\ x_{n-1} \neq y_{n-1}}}\left|\sum_{x_{n} \in \mathcal{X}_{n}} \mathbf{e}_{p}\left(x_{n}\left(x_{1}-y_{1}\right) \ldots\left(x_{n-1}-y_{n-1}\right)\right)\right|,
$$

by Lemma 2.1 it is sufficient to show that

$$
S^{2} \leqslant p X_{n}\left(D_{n-1}^{\times, *}\left(\mathcal{X}_{1}\right) \ldots D_{n-1}^{\times, *}\left(\mathcal{X}_{n-1}\right)\right)^{1 /(n-1)} .
$$

Let $I(\lambda)$ count the number of solutions to the equation

$$
\lambda=\left(x_{1}-y_{1}\right) \ldots\left(x_{n-1}-y_{n-1}\right), \quad x_{i}, y_{i} \in \mathcal{X}_{i}, \quad x_{i} \neq y_{i},
$$

so that

$$
S=\sum_{\lambda} I(\lambda)\left|\sum_{x_{n} \in \mathcal{X}_{n}} e_{p}\left(\lambda x_{1}\right)\right|
$$

and hence by Lemma 2.4

$$
S^{2} \leqslant\left(\sum_{\lambda} I(\lambda)^{2}\right) p X_{n}
$$

and the result follows from Lemma 2.2 since

$$
\sum_{\lambda} I(\lambda)^{2}=D_{n-1}^{\times}\left(\mathcal{X}_{1}, \ldots, \mathcal{X}_{n-1}\right) .
$$

Our next estimate does better in applications over Lemma 2.5 when our sets have $\mathcal{X}_{1}, \ldots, \mathcal{X}_{n}$ have large cardinalities.

Lemma 2.6. Let $n \geqslant 2$. Suppose $S\left(\mathcal{X}_{1}, \ldots, \mathcal{X}_{n} ; \omega_{1}, \ldots, \omega_{n}\right)$ is defined as in (1.1). Then we have

$$
\begin{gathered}
\left|S\left(\mathcal{X}_{1}, \ldots, \mathcal{X}_{n} ; \omega_{1}, \ldots, \omega_{n}\right)\right|^{2^{n}} \ll\left(X_{1} \ldots X_{n}\right)^{2^{n}}\left(\frac{1}{X_{1}^{2^{n-1}}}+\cdots+\frac{1}{X_{n}}\right) \\
+p^{1 / 2}\left(X_{1} \ldots X_{n}\right)^{2^{n}-2}\left(\widetilde{D}_{n}^{\times, *}\left(\mathcal{X}_{1}\right) \ldots \widetilde{D}_{n}^{\times, *}\left(\mathcal{X}_{n}\right)\right)^{1 / 2 n} .
\end{gathered}
$$

Proof. Writing

$$
S=\sum_{\substack{x_{2}, y_{2} \in \mathcal{X}_{2} \\ x_{2} \neq y_{2}}} \ldots \sum_{\substack{x_{n}, y_{n} \in \mathcal{X}_{n} \\ x_{n} \neq y_{n}}}\left|\sum_{x_{1} \in \mathcal{X}_{1}} \mathbf{e}_{p}\left(x_{1}\left(x_{2}-y_{2}\right) \ldots\left(x_{n}-y_{n}\right)\right)\right|,
$$


by Lemma 2.1 it is sufficient to show that

$$
\begin{aligned}
S^{2} \leqslant & \frac{\left(X_{1} \ldots X_{n}\right)^{4}}{X_{1}^{2}} \\
& +\left(X_{2} \ldots X_{n}\right)^{2} p^{1 / 2}\left(\widetilde{D}_{n}^{\times, *}\left(\mathcal{X}_{1}\right) \ldots \widetilde{D}_{n}^{\times, *}\left(\mathcal{X}_{n}\right)\right)^{1 / 2 n} .
\end{aligned}
$$

Applying the Cauchy-Schwarz inequality, interchanging summation and isolating the diagonal contribution gives

$$
S^{2} \leqslant X_{1}\left(X_{2} \ldots X_{n}\right)^{4}+\left(X_{2} \ldots X_{n}\right)^{2}\left|\sum_{\lambda=1}^{p-1} I(\lambda) e_{p}(\lambda)\right|
$$

where $I(\lambda)$ counts the number of solutions to the equation

$$
\left(x_{1}-y_{1}\right) \ldots\left(x_{n}-y_{n}\right)=\lambda, \quad x_{i}, y_{i} \in \mathcal{X}_{i}, \quad x_{i} \neq y_{i} .
$$

Let

$$
\Delta=\frac{X_{1}\left(X_{1}-1\right) \ldots X_{n}\left(X_{n}-1\right)}{p-1}
$$

and write

$$
\sum_{\lambda=1}^{p-1} I(\lambda) e_{p}(\lambda)=\Delta \sum_{\lambda=1}^{p-1} e_{p}(\lambda)+\sum_{\lambda=1}^{p-1}(I(\lambda)-\Delta) e_{p}(\lambda)
$$

We have

$$
\left|\sum_{\lambda=1}^{p-1} I(\lambda) e_{p}(\lambda)\right| \ll \frac{\left(X_{1} \ldots X_{n}\right)^{2}}{p}+\sum_{\lambda=1}^{p-1}|I(\lambda)-\Delta| .
$$

With notation as in Lemma 2.3, by the Cauchy-Schwarz inequality

$$
\sum_{\lambda=1}^{p-1}|I(\lambda)-\Delta| \leqslant p^{1 / 2}\left(\sum_{\lambda=1}^{p-1}|I(\lambda)-\Delta|^{2}\right)^{1 / 2}=p^{1 / 2} \widetilde{D}_{n}^{\times, *}\left(\mathcal{X}_{1}, \ldots, \mathcal{X}_{n}\right)^{1 / 2},
$$

and hence

$$
\sum_{\lambda=1}^{p-1}|I(\lambda)-\Delta| \leqslant p^{1 / 2}\left(\widetilde{D}_{n}^{\times, *}\left(\mathcal{X}_{1}\right) \ldots \widetilde{D}_{n}^{\times, *}\left(\mathcal{X}_{n}\right)\right)^{1 / 2 n}
$$

Combining the above with (2.1) and (2.2) gives

$$
\begin{aligned}
S^{2} \leqslant & \frac{\left(X_{1} \ldots X_{n}\right)^{4}}{X_{1}^{3}}+\frac{\left(X_{1} \ldots X_{n}\right)^{4}}{p} \\
& +\left(X_{2} \ldots X_{n}\right)^{2} p^{1 / 2}\left(\widetilde{D}_{n}^{\times, *}\left(\mathcal{X}_{1}\right) \ldots \widetilde{D}_{n}^{\times, *}\left(\mathcal{X}_{n}\right)\right)^{1 / 2 n} \\
& \ll \frac{\left(X_{1} \ldots X_{n}\right)^{4}}{X_{1}^{3}}+\left(X_{2} \ldots X_{n}\right)^{2} p^{1 / 2}\left(\widetilde{D}_{n}^{\times, *}\left(\mathcal{X}_{1}\right) \ldots \widetilde{D}_{n}^{\times, *}\left(\mathcal{X}_{n}\right)\right)^{1 / 2 n},
\end{aligned}
$$

and completes the proof. 
2.2. Estimates for $D_{k}^{\times}(\mathcal{A})$. In this section we give estimates for $D_{k}^{\times}(\mathcal{A})$ which will be combined with results from Section 2.1 to obtain estimates for multilinear sums. We first recall the following result [26, Theorem 32].

Lemma 2.7. Suppose $\mathcal{A} \subset \mathbb{F}_{p}$ is a set and $|\mathcal{A}|=A$. For all $k \geqslant 2$

$$
D_{k}^{\times}(\mathcal{A})-\frac{A^{4 k}}{p} \ll_{k}(\log A)^{4} A^{4 k-2-2^{-k+2}} E^{+}(\mathcal{A})^{1 / 2^{k-1}} .
$$

We then have the following lemma [26, Theorem 41].

Lemma 2.8. Let $\mathcal{A} \subset \mathbb{F}_{p}$ be a set, $A \leqslant p^{2846 / 4991}$. Then for any $c<\frac{1}{434}$ one has

$$
D_{2}^{\times}(\mathcal{A}) \ll A^{13 / 2-c} .
$$

Furthermore, if $A \leqslant p^{48 / 97}$, then for any $c_{1}<\frac{1}{192}$ one has

$$
D_{2}^{\times}(\mathcal{A}) \ll A^{13 / 2-c_{1}} .
$$

We first notice that from the proof of [26, Theorem 32] we have

$$
D_{k}^{\times}(\mathcal{A})-\frac{A^{4 k}}{p} \ll_{k}(\log A)^{2} A^{2 k+1}\left(D_{k-1}^{\times}(A)-\frac{A^{4(k-1)}}{p}\right)^{1 / 2} .
$$

Using $E^{+}(\mathcal{A}) \leqslant A^{3}$, combined with Lemma 2.8 and (2.3) we have the following corollary.

Corollary 2.9. Suppose $\mathcal{A} \subset \mathbb{F}_{p}$ is a set and $|\mathcal{A}|=A$. For all $k \geqslant 2$

$$
D_{k}^{\times}(\mathcal{A})-\frac{A^{4 k}}{p} \ll_{k}(\log A)^{4} A^{4 k-2+2^{-k+1}} .
$$

Similarly if $A \leqslant p^{2846 / 4991}$, for any $c<\frac{1}{434}$ we have

$$
D_{k}^{\times}(\mathcal{A})-\frac{A^{4 k}}{p} \ll_{k}(\log A)^{4} A^{4 k-2+2^{-k+1}-c 2^{-k+2}}
$$

and if $A \leqslant p^{48 / 97}$, for any $c_{1}<\frac{1}{192}$ we have

$$
D_{k}^{\times}(\mathcal{A})-\frac{A^{4 k}}{p} \ll_{k}(\log A)^{4} A^{4 k-2+2^{-k+1}-c_{1} 2^{-k+2}} .
$$

It is clear that we can use the above to give other estimates on $D_{k}^{\times}$ using previous estimates on $D_{2}^{\times}$. We recall the following result [18, Lemma 2.6], which is given from Murphy et. al [21] result on collinear triples. 
Lemma 2.10. Let $\mathcal{A} \subset \mathbb{F}_{p}$. Then

$$
D_{2}^{\times}(\mathcal{A})-\frac{A^{8}}{p} \ll p^{1 / 2} A^{11 / 2} .
$$

Again, we have the following corollary.

Corollary 2.11. Let $\mathcal{A} \subset \mathbb{F}_{p}$. Then

$$
D_{k}^{\times}(\mathcal{A})-\frac{A^{4 k}}{p} \ll_{k} p^{2^{1-k}}(\log A)^{4} A^{4 k-2-2^{-k+1}} .
$$

We next prepare to give an estimate for $D_{k}^{\times}(\mathcal{A})$ which improves on the above results for sets of cardinality a little larger than $p^{1 / 2}$. As in Shkredov [26], our main tool is Rudnev's point plane incidence bound [23].

Lemma 2.12. Let $p$ be an odd prime, $\mathcal{P} \subset \mathbb{F}_{p}^{3}$ a set of points and $\Pi$ a collection of planes in $\mathbb{F}_{p}^{3}$. Suppose $|\mathcal{P}| \leqslant|\Pi|$ and that $k$ is the maximum number of collinear points in $\mathcal{P}$. Then the number of pointplanes incidences satisfies

$$
\mathcal{I}(\mathcal{P}, \Pi) \leqslant \frac{|\mathcal{P}||\Pi|}{p}+|\mathcal{P}|^{1 / 2}|\Pi|+k|\mathcal{P}| .
$$

Lemma 2.13. For a prime number $p$ and a subset $\mathcal{A} \subseteq \mathbb{F}_{p}$ with $|\mathcal{A}|=A$ we have

$$
\begin{aligned}
D_{2}^{\times}(\mathcal{A})=\frac{A^{8}}{p} & +O\left(A^{6}(\log A)^{2}+p^{1 / 2} A^{4} E_{+}(\mathcal{A})^{1 / 2}(\log A)^{2}\right) \\
& +O\left(p A^{4}(\log A)^{2}\right) .
\end{aligned}
$$

Proof. We have

$$
D_{2}^{\times}(\mathcal{A})=\sum_{\substack{a_{i} \in \mathcal{A} \\\left(a_{1}-a_{2}\right)\left(a_{3}-a_{4}\right)=\left(a_{5}-a_{6}\right)\left(a_{7}-a_{8}\right) \\ a_{5} \neq a_{6}}} 1+O\left(A^{6}\right) .
$$

Let $I(x)$ denote the indicator function of the multiset

$$
\left\{a-a^{\prime}: a, a^{\prime} \in \mathcal{A}\right\}
$$

and let $\widehat{I}$ denote the Fourier transform of $I$. We note that the Fourier coefficients satisfy

$$
\widehat{I}(x)=\left|\sum_{a \in \mathcal{A}} e_{p}(a x)\right|^{2}
$$


We have

$$
\begin{aligned}
D_{2}^{\times}(A) & =\sum_{\substack{a_{i} \in \mathcal{A} \\
a_{5} \neq a_{6}}} I\left(\frac{\left(a_{1}-a_{2}\right)\left(a_{3}-a_{4}\right)}{\left(a_{5}-a_{6}\right)}\right)+O\left(A^{6}\right) \\
& =\frac{A^{8}}{p}+O\left(A^{6}\right)+W
\end{aligned}
$$

where

$$
W=\frac{1}{p} \sum_{y=1}^{p-1} \widehat{I}(y) \sum_{\substack{a_{i} \in \mathcal{A} \\ a_{5} \neq a_{6}}} e_{p}\left(-y\left(a_{1}-a_{2}\right)\left(a_{3}-a_{4}\right)\left(a_{5}-a_{6}\right)^{-1}\right) .
$$

We have

$$
\begin{aligned}
W & \leqslant \frac{1}{p} \sum_{y=1}^{p-1} \sum_{z=1}^{p} \widehat{I}(y) \widehat{I}(z) \sum_{\substack{a_{i} \in \mathcal{A} \\
y=\left(a_{3}-a_{4}\right) z \\
a_{3} \neq a_{4}}} 1 \\
& =\frac{A^{5}}{p} \sum_{y=1}^{p-1} \widehat{I}(y)+\frac{1}{p} \sum_{y=1}^{p-1} \sum_{z=1}^{p-1} \widehat{I}(y) \widehat{I}(z) \sum_{\substack{a_{i} \in \mathcal{A} \\
\left(a_{1}-a_{2}\right) y=\left(a_{3}-a_{4}\right) z}} 1
\end{aligned}
$$

where we have removed the condition $a_{3} \neq a_{4}$ in the last display since by (2.4) the Fourier coefficients are nonnegative. The above implies

$$
W \leqslant W_{0}+O\left(A^{6}\right)
$$

where

$$
W_{0}=\frac{1}{p} \sum_{y=1}^{p-1} \sum_{z=1}^{p-1} \widehat{I}(y) \widehat{I}(z) \sum_{\substack{a_{i} \in \mathcal{A} \\\left(a_{1}-a_{2}\right) y=\left(a_{3}-a_{4}\right) z}} 1 .
$$

For integer $i \geqslant 1$ we define the sets

$$
J(i)=\left\{1 \leqslant z \leqslant p: 2^{i-1}-1 \leqslant \widehat{I}(z)<2^{i}-1\right\},
$$

so that

$$
W_{0} \ll \frac{1}{p} \sum_{1 \leqslant i, j \ll \log A} 2^{i+j} W(i, j)
$$

where

$$
W(i, j)=\sum_{\substack{a_{i} \in \mathcal{A}, y \in J(i), z \in J(j) \\\left(a_{1}-a_{2}\right) y=\left(a_{3}-a_{4}\right) z}} 1
$$


Fix some pair $(i, j)$ and consider $W(i, j)$. If $|J(i)| \leqslant|J(j)|$, then we consider the set of points

$$
\mathcal{P}=\left\{\left(a_{1} y, y, a_{3}\right): y \in J(i), a_{1}, a_{3} \in \mathcal{A}\right\},
$$

and the collection of planes

$$
\Pi=\left\{x_{1}-a_{2} x_{2}-z x_{3}+a_{4} z=0: z \in J(j), \quad a_{2}, a_{4} \in \mathcal{A}\right\} .
$$

We see that $W(i, j)$ is bounded by the number of point-plane incidences between $\mathcal{P}$ and $\Pi$

$$
W(i, j) \leqslant \mathcal{I}(\mathcal{P}, \Pi) .
$$

Since the maximum number of collinear points in $\mathcal{P}$ is $\max \{A,|J(i)|\}$ an application of Lemma 2.12 gives

$$
\begin{aligned}
W(i, j) \ll \frac{A^{4}|J(i)||J(j)|}{p}+A^{3}|J(i)|^{1 / 2}|J(j)| & \\
& +A^{2}|J(i)| \max \{A,|J(i)|\} .
\end{aligned}
$$

In a similar fashion, if $|J(j)| \leqslant|J(i)|$ then

$$
\begin{aligned}
W(i, j) \ll \frac{A^{4}|J(i)||J(j)|}{p}+A^{3}|J(j)|^{1 / 2}|J(i)| & \\
& +A^{2}|J(j)| \max \{A,|J(j)|\} .
\end{aligned}
$$

This implies that

$$
\begin{aligned}
W(i, j) \ll & \frac{A^{4}|J(i)||J(j)|}{p}+A^{3}|J(i)|^{1 / 2}|J(j)|+A^{3}|J(j)|^{1 / 2}|J(i)| \\
& +A^{2} \min \left\{|J(i)|^{2},|J(j)|^{2}\right\} \\
\ll & \frac{A^{4}|J(i)||J(j)|}{p}+A^{3}|J(i)|^{1 / 2}|J(j)|+A^{3}|J(j)|^{1 / 2}|J(i)| \\
& +A^{2}|J(i)||J(j)|,
\end{aligned}
$$

and hence substituting the above into (2.8) we get

$$
\begin{aligned}
W_{0} \ll \frac{A^{4}}{p^{2}} & \left.\sum_{1 \leqslant i \ll \log A} 2^{i}|J(i)|\right)^{2} \\
+ & \frac{A^{3}}{p}\left(\sum_{1 \leqslant i \ll \log A} 2^{i}|J(i)|^{1 / 2}\right)\left(\sum_{1 \leqslant i \ll \log A} 2^{i}|J(i)|\right) \\
& +\frac{A^{2}}{p}\left(\sum_{1 \leqslant i \ll \log A} 2^{i}|J(i)|\right)^{2} .
\end{aligned}
$$


Recalling (2.4) and (2.7), we have

$$
\begin{aligned}
\sum_{1 \leqslant i \ll \log A} 2^{i}|J(i)| & \ll p+\sum_{2 \leqslant i \ll \log A} 2^{i}|J(i)| \\
& \ll p+\log A \sum_{y=1}^{p}\left|\sum_{a \in \mathcal{A}} e_{p}(y a)\right|^{2}=p A \log A,
\end{aligned}
$$

and

$$
\begin{aligned}
\left(\sum_{1 \leqslant i \ll \log A} 2^{i}|J(i)|^{1 / 2}\right)^{2} & \ll p+\log A \sum_{2 \leqslant i \ll \log A} 2^{2 i}|J(i)| \\
& \ll p+(\log A)^{2} \sum_{y=1}^{p}\left|\sum_{a \in \mathcal{A}} e_{p}(y a)\right|^{4},
\end{aligned}
$$

so that

$$
\sum_{1 \leqslant i \ll \log A} 2^{i}|J(i)|^{1 / 2} \ll p^{1 / 2} E_{+}(\mathcal{A})^{1 / 2} \log A .
$$

This implies

$$
W \ll A^{6}(\log A)^{2}+p^{1 / 2} A^{4} E_{+}(\mathcal{A})^{1 / 2}(\log A)^{2}+p A^{4},
$$

and hence by (2.5) and (2.6)

$$
\begin{aligned}
D_{2}^{\times}(A) & =\frac{A^{8}}{p}+O\left(A^{6}(\log A)^{2}\right)+O\left(p^{1 / 2} A^{4} E_{+}(\mathcal{A})^{1 / 2}(\log A)^{2}\right) \\
& +O\left(p A^{4}(\log A)^{2}\right),
\end{aligned}
$$

which completes the proof.

We next establish a recurrence type inequality similar to [26, Theorem 32].

Lemma 2.14. For a prime number $p$ and a subset $\mathcal{A} \subseteq \mathbb{F}_{p}$ with $|\mathcal{A}|=A$ we have

$$
D_{k}^{\times}(\mathcal{A})=\frac{A^{4 k}}{p}+O_{k}\left(\left(A^{4 k-2}+p A^{4 k-4}+p^{1 / 2} A^{2 k} D_{k-1}^{\times}(\mathcal{A})^{1 / 2}\right) \log ^{2} A\right) .
$$

Proof. Let $D_{k}^{\prime}(\mathcal{A})$ count the number of solutions to the equation

$$
\left(a_{1,1}-a_{1,2}\right) \ldots\left(a_{k, 1}-a_{k, 2}\right)=\left(a_{k+1,1}-a_{k+1,2}\right) \ldots\left(a_{2 k, 1}-a_{2 k, 2}\right),
$$

with variables $a_{1,1}, \ldots, a_{2 k, 2} \in \mathcal{A}$ satisfying

$$
a_{1,1} \neq a_{1,2}, \quad a_{k+1,1} \neq a_{k+1,2}
$$

so that

$$
D_{k}^{\times}(\mathcal{A})=D_{k}^{\prime}(\mathcal{A})+O\left(A^{4 k-2}\right) .
$$


Let $I(y)$ denote the indicator function of the multiset

$$
\left\{\left(a_{2,1}-a_{2,2}\right) \ldots\left(a_{k, 1}-a_{k, 2}\right): a_{2,1}, \ldots, a_{k, 2} \in \mathcal{A}\right\},
$$

and let $\widehat{I}(y)$ denote the Fourier transform of $I$. We have

$$
\begin{aligned}
D_{k}^{\prime} & (\mathcal{A})=\sum_{\substack{a_{j, 1}, a_{j, 2} \in \mathcal{A} \\
a_{1,1} \neq a_{1,2} \\
a_{k+1,1} \neq a_{k+1,2}}} I\left(\left(a_{k+1,1}-a_{k+1,2}\right) \ldots\left(a_{2 k, 1}-a_{2 k, 2}\right)\left(a_{1,1}-a_{1,2}\right)^{-1}\right) \\
= & \frac{1}{p} \sum_{y=1}^{p-1} \widehat{I}(y) \\
& \sum_{\substack{a_{j, 1}, a_{j, 2} \in \mathcal{A} \\
a_{1,1} \neq \neq}}^{a_{k+1,2}} e_{p}\left(-y\left(a_{k+1,1}-a_{k+1,2}\right) \ldots\left(a_{2 k, 1}-a_{2 k, 2}\right)\left(a_{1,1}-a_{1,2}\right)^{-1}\right) \\
= & \frac{1}{p} \sum_{z=1}^{p} \sum_{y=1}^{p-1} \widehat{I}(y) \widehat{I}(-z) \sum_{\substack{a_{i, j} \in \mathcal{A} \\
y\left(a_{1,1}-a_{1,2}\right)=z\left(a_{2,1}-a_{2,2}\right) \\
a_{j, 1} \neq a_{j, 2}, j=1,2}}^{p} 1,
\end{aligned}
$$

which implies that

$$
D_{k}^{\prime}(\mathcal{A})=\frac{A^{4 k}}{p}+W_{0}+O\left(A^{4 k-2}\right)
$$

where

$$
W_{0}=\frac{1}{p} \sum_{z=1}^{p-1} \sum_{y=1}^{p-1} \widehat{I}(y) \widehat{I}(-z) \sum_{\substack{a_{i, j} \in \mathcal{A} \\\left(a_{1,1}-a_{1,2}\right)=z\left(a_{2,1}-a_{2,2}\right) \\ a_{j, 1} \neq a_{j, 2}, j=1,2}} 1 .
$$

For integer $i \geqslant 1$ we define

$$
J(i)=\left\{y \in \mathbb{F}_{p}^{*}: 2^{i-1}-1 \leqslant|\widehat{I}(y)| \leqslant 2^{i}-1\right\},
$$

so that

$$
W_{0} \ll \frac{1}{p} \sum_{i, j \ll \log A^{2 k}} 2^{i+j} W(i, j)
$$

where

$$
W(i, j)=\sum_{\substack{a_{i, j} \in \mathcal{A}, y \in J(i), z \in J(j) \\ y\left(a_{1,1}-a_{1,2}\right)=z\left(a_{2,1}-a_{2,2}\right) \\ a_{j, 1} \neq a_{j, 2}, j=1,2}} 1 .
$$


Using Lemma 2.12 as in the proof of Lemma 2.13, we see that

$$
\begin{gathered}
W(i, j) \ll \frac{A^{4}|J(i)||J(j)|}{p}+A^{3}|J(i)|^{1 / 2}|J(j)|+A^{3}|J(j)|^{1 / 2}|J(i)| \\
+A^{2}|J(i)||J(j)| .
\end{gathered}
$$

We have

$$
\begin{aligned}
& \sum_{i \ll \log A} 2^{i}|J(i)| \\
& \ll p+\sum_{y=1}^{p-1}\left|\sum_{\substack{a_{i, 1}, a_{i, 2} \in \mathcal{A} \\
1 \leqslant i \leqslant k-1}} e_{p}\left(y\left(a_{1,1}-a_{1,2}\right) \ldots\left(a_{k-1,1}-a_{k-1,2}\right)\right)\right| \\
& \leqslant p+\sum_{\substack{a_{i, 1}, a_{i, 2} \in \mathcal{A} \\
2 \leqslant i \leqslant k-1}}^{p \sum_{y=1}^{p-1} \mid}\left|\sum_{a \in A} e_{p}\left(y\left(a_{2,1}-a_{2,2}\right) \ldots\left(a_{k-1,1}-a_{k-1,2}\right) a\right)\right|^{2} \\
& \ll p A^{2 k-3},
\end{aligned}
$$

and

$$
\begin{aligned}
& \sum_{i \ll \log A} 2^{i}|J(i)|^{1 / 2} \ll p^{1 / 2} \\
& +\left(\log A \sum_{y=1}^{p-1}\left|\sum_{\substack{a_{i, 1}, a_{i, 2} \in \mathcal{A} \\
1 \leqslant i \leqslant k-1}} e_{p}\left(y\left(a_{1,1}-a_{1,2}\right) \ldots\left(a_{k-1,1}-a_{k-1,2}\right)\right)\right|^{2}\right)^{1 / 2}
\end{aligned}
$$

so that

$$
\sum_{i \ll \log A} 2^{i}|J(i)|^{1 / 2} \ll_{k}(\log A)^{1 / 2} p^{1 / 2} D_{k-1}^{\times}(\mathcal{A})^{1 / 2} .
$$

Combining the above with (2.13) and (2.14) we see that

$$
W_{0} \ll_{k}\left(A^{4 k-2}+p A^{4 k-4}+p^{1 / 2} A^{2 k} D_{k-1}^{\times}(\mathcal{A})^{1 / 2}\right) \log ^{2} A,
$$

and hence by (2.11) and (2.12)

$$
\begin{aligned}
D_{k}^{\times}(A)= & \frac{A^{4 k}}{p} \\
& +O_{k}\left(\left(A^{4 k-2}+p A^{4 k-4}+p^{1 / 2} A^{2 k} D_{k-1}^{\times}(\mathcal{A})^{1 / 2}\right) \log ^{2} A\right),
\end{aligned}
$$

which completes the proof.

Combining Lemma 2.13 and Lemma 2.14 with an induction argument gives the following Corollary. 
Corollary 2.15. For a prime number $p$ and a subset $\mathcal{A} \subseteq \mathbb{F}_{p}$ with $|\mathcal{A}|=A \geqslant p^{1 / 2}$ we have

$$
\begin{aligned}
& D_{k}^{\times}(\mathcal{A})=\frac{A^{4 k}}{p} \\
& \quad+O_{k}\left(\left(A^{4 k-2}+p^{1-2^{-(k-1)}} A^{4 k-4} E_{+}(\mathcal{A})^{2^{-(k-1)}}\right) \log ^{4} A\right) .
\end{aligned}
$$

Using the trivial bound $E_{+}(\mathcal{A}) \leqslant A^{3}$ in Corollary 2.15 gives the following sharp asymptotic formula for $D_{k}^{\times}(\mathcal{A})$ for sets of cardinality a little larger than $p^{1 / 2}$.

Corollary 2.16. For any $k \geqslant 3$ and $A \geqslant p^{1 / 2+1 /\left(2^{k+1}-6\right)}$ we have

$$
D_{k}^{\times}(\mathcal{A})=\frac{A^{4 k}}{p}+O_{k}\left(A^{4 k-2} \log ^{4} A\right) .
$$

We define $N(\mathcal{X}, \mathcal{Y}, \mathcal{Z})$ to be the number of solutions to

$$
x_{1}\left(y_{1}-z_{1}\right)=x_{2}\left(y_{2}-z_{2}\right)
$$

with $x_{1}, x_{2} \in \mathcal{X}, y_{1}, y_{2} \in \mathcal{Y}$ and $z_{1}, z_{2} \in \mathcal{Z}$. We now recall [22, Corollary $2.4]$.

Lemma 2.17. Let $\mathcal{X}, \mathcal{Y}, \mathcal{Z} \subset \mathbb{F}_{p}^{*}$ with $|\mathcal{X}|=X,|\mathcal{Y}|=Y,|\mathcal{Z}|=Z$ and $M=\max (X, Y, Z)$. Then

$$
N(\mathcal{X}, \mathcal{Y}, \mathcal{Z}) \ll \frac{X^{2} Y^{2} Z^{2}}{p}+X^{3 / 2} Y^{3 / 2} Z^{3 / 2}+M X Y Z
$$

\subsection{Proof of Theorem 1.1.}

Proof. Let

$$
S=S\left(\mathcal{X}_{1}, \ldots, \mathcal{X}_{n} ; \omega_{1}, \ldots, \omega_{n}\right) .
$$

By Lemma 2.1, after permuting the variables, we have

$$
\begin{aligned}
&|S|^{2^{n-1}} \ll\left(X_{1} \ldots X_{n}\right)^{2^{n-1}}\left(\frac{1}{X_{n-1}}+\cdots+\frac{1}{X_{1}^{2^{n-2}}}\right) \\
&+\left(X_{1} \ldots X_{n-1}\right)^{2^{n-1}-2} X_{n}^{2^{n-1}-1} \sum_{\substack{x_{1}, y_{1} \in \mathcal{X}_{1} \\
x_{1} \neq y_{1}}} \ldots \sum_{\substack{x_{n-1}, y_{n-1} \in \mathcal{X}_{n-1} \\
x_{n-1} \neq y_{n-1}}} \\
& \qquad\left|\sum_{x_{n} \in \mathcal{X}_{n}} \mathbf{e}_{p}\left(x_{n}\left(x_{1}-y_{1}\right) \ldots\left(x_{n-1}-y_{n-1}\right)\right)\right| .
\end{aligned}
$$

We now collect together $\left(x_{2}-y_{2}\right) \ldots\left(x_{n-1}-y_{n-1}\right)=\lambda$ and denote the number of solutions to this equation to be $J(\lambda)$. Similarly we collect 
$x_{1}\left(x_{n}-y_{n}\right)=\mu$ and we denote the number of solutions to this equation to be $I(\mu)$. Hence,

$$
\begin{aligned}
|S|^{2^{n-1}} \ll_{n}( & \left.X_{1} \ldots X_{n}\right)^{2^{n-1}}\left(\frac{1}{X_{n-1}}+\cdots+\frac{1}{X_{1}^{2^{n-2}}}\right) \\
& +\left(X_{1} \ldots X_{n-1}\right)^{2^{n-1}-2} X_{n}^{2^{n-1}-1} \sum_{\lambda \in \mathbb{F}_{p}^{*}} J(\lambda)\left|\sum_{\mu \in \mathbb{F}_{p}} I(\mu) \mathbf{e}_{p}(\lambda \mu)\right| \\
= & \left(X_{1} \ldots X_{n}\right)^{2^{n-1}}\left(\frac{1}{X_{n-1}}+\cdots+\frac{1}{X_{1}^{2^{n-2}}}\right) \\
& +\left(X_{1} \ldots X_{n-1}\right)^{2^{n-1}-2} X_{n}^{2^{n-1}-1} \sum_{\lambda \in \mathbb{F}_{p}^{*}} \sum_{\mu \in \mathbb{F}_{p}} J(\lambda) \eta_{\lambda} I(\mu) \mathbf{e}_{p}(\lambda \mu)
\end{aligned}
$$

for some complex weight $\eta_{\lambda}$ with $\left|\eta_{\lambda}\right|=1$. Now, by Lemma 2.17 with $X=Y=X_{1}, Z=X_{n}$ we have

$$
\sum_{\mu \in \mathbb{F}_{p}} I(\mu)^{2}=N\left(\mathcal{X}_{n}, \mathcal{X}_{1}, \mathcal{X}_{1}\right) \ll X_{1}^{3} X_{n}^{3 / 2}
$$

Similarly,

$$
\sum_{\lambda \in \mathbb{F}_{p}^{*}} J(\lambda)^{2}=D_{n-2}^{\times, *}\left(\mathcal{X}_{2}, \ldots, \mathcal{X}_{n-1}\right) .
$$

We apply Corollary 2.9 and 2.11 combined with Lemma 2.2 along with Lemma 2.4 to obtain

$$
\begin{aligned}
|S|^{2^{n-1}} \ll_{n}\left(X_{1} \ldots X_{n}\right)^{2^{n-1}} & \left(\frac{1}{X_{n-1}}+\cdots+\frac{1}{X_{1}^{2^{n-2}}}\right) \\
& +\left(X_{1} \ldots X_{n}\right)^{2^{n-1}} p^{1 / 2} X_{1}^{-1 / 2} X_{n}^{-1 / 4}\left(\prod_{i=2}^{n-1} B_{n}\left(\mathcal{X}_{i}\right)^{2^{n-1}}\right)
\end{aligned}
$$

This completes the proof.

2.4. Proof of Theorem 1.2. We note that the conditions (1.6) and Corollary 2.16 imply that

$$
\widetilde{D}_{n}^{\times, *}\left(\mathcal{X}_{i}\right) \ll(\log p)^{4} X_{i}^{4 n-2},
$$

and hence by Lemma 2.6

$$
\begin{aligned}
\mid S\left(\mathcal{X}_{1}, \ldots,\right. & \left.\mathcal{X}_{n} ; \omega_{1}, \ldots, \omega_{n}\right)\left.\right|^{2^{n}} \ll\left(X_{1} \ldots X_{n}\right)^{2^{n}}\left(\frac{1}{X_{1}^{2^{n-1}}}+\cdots+\frac{1}{X_{n}}\right) \\
& +(\log p)^{4} p^{1 / 2}\left(X_{1} \ldots X_{n}\right)^{2^{n}-1 / n}
\end{aligned}
$$

from which the desired result follows. 


\section{Multinomial Exponential Sums}

3.1. Preliminaries. The aim of this section is to extend the results of [17] and [19] beyond the cases of trinomials and quadrinomials, to more general multinomial sums.

We recall the following bound of [19].

Lemma 3.1. Let $\mathcal{G} \subseteq \mathbb{F}_{p}^{*}$ be a multiplicative subgroup with $|\mathcal{G}|=G$. Then

$$
D_{2}^{\times}(\mathcal{G})-\frac{G^{8}}{p} \ll \begin{cases}p^{1 / 2} G^{\frac{11}{2}}, & \text { if } G \geqslant p^{\frac{2}{3}} \\ G^{7} p^{-\frac{1}{2}}, & \text { if } p^{\frac{2}{3}}>G \geqslant p^{\frac{1}{2}} \log p \\ G^{6} \log G, & \text { if } G<p^{\frac{1}{2}} \log p .\end{cases}
$$

Combining with (2.3) and observing which term dominates we get the following corollary.

Corollary 3.2. Let $\mathcal{G} \subseteq \mathbb{F}_{p}^{*}$ be a multiplicative subgroup with $|\mathcal{G}|=G$. Then

$$
D_{k}^{\times}(\mathcal{G}) \ll \begin{cases}G^{4 k} p^{-1} & \text { if } G \geqslant p^{\frac{1}{2}} \log p, \\ G^{4 k-2+o(1)}, & \text { if } G<p^{\frac{1}{2}} \log p .\end{cases}
$$

We also have the following result as a consequence of [17, Lemma $2.4]$.

Lemma 3.3. Let $\mathcal{G}, \mathcal{H} \subset \mathbb{F}_{p}^{*}$ be multiplicative subgroups with cardinalities $G, H$ respectively with $G \geqslant H$. Then,

$$
N(\mathcal{H}, \mathcal{G}, \mathcal{G}) \ll \begin{cases}H^{2} G^{\frac{7}{2}} p^{-\frac{1}{2}} & \text { if } G \geqslant p^{\frac{1}{2}} \log p, \\ H^{2} G^{\frac{5}{2}+o(1)}, & \text { if } G<p^{\frac{1}{2}} \log p .\end{cases}
$$

We then have the following result on multilinear exponential sums over subgroups, which may be of independent interest to the reader.

Lemma 3.4. Let $\mathcal{X}_{i} \subset \mathbb{F}_{p}$ be multiplicative subgroups with $\left|\mathcal{X}_{i}\right|=X_{i}$, $X_{1} \geqslant X_{2} \geqslant \cdots \geqslant X_{n}, n \geqslant 4$. Then

$$
\begin{aligned}
S\left(\mathcal{X}_{1}, \ldots, \mathcal{X}_{n} ; \omega_{1}, \ldots, \omega_{n}\right) \ll_{n}( & \left.X_{1} \ldots X_{n}\right) p^{\frac{1}{2^{n}}} A_{n}\left(\mathcal{X}_{1}\right) \prod_{i=2}^{n-1} B_{n}\left(\mathcal{X}_{i}\right) \\
& +\left(X_{1} \ldots X_{n}\right)\left(\frac{1}{X_{n}^{1 / 2}}+\cdots+\frac{1}{X_{1}^{1 / 2^{n}}}\right)
\end{aligned}
$$

where

$$
A_{n}\left(\mathcal{X}_{1}\right)= \begin{cases}X_{1}^{-\frac{1}{2^{n+1}}} p^{-\frac{1}{2^{n+1}}}, & \text { if } X_{1} \geqslant p^{\frac{1}{2}} \log p \\ X_{1}^{-\frac{3}{2^{n+1}}+o(1)}, & \text { if } X_{1}<p^{\frac{1}{2}} \log p\end{cases}
$$


and

$$
B_{n}\left(\mathcal{X}_{i}\right)= \begin{cases}p^{-\frac{1}{2^{n}(n-2)}}, & \text { if } X_{i} \geqslant p^{\frac{1}{2}} \log p, \\ X_{i}^{-\frac{1}{2^{n-1}(n-2)}}, & \text { if } X_{i}<p^{\frac{1}{2}} \log p .\end{cases}
$$

Proof. The proof follows that of Theorem 1.1, however we use Corollary 3.2 and Lemma 3.3 in place of their relevant results on arbitrary sets.

3.2. Proof of Theorem 1.3. Let $\alpha_{k_{i}}=\operatorname{gcd}\left(k_{i}, p-1\right)$ for each $i=$ $1, \ldots, t$. We then let $\mathcal{G}_{\alpha_{i}}$ be the subgroups of $\mathbb{F}_{p}^{*}$ generated by the elements of order $\alpha_{k_{i}}$. Then

$$
\begin{aligned}
& T_{\chi}(\Psi)=\frac{1}{\alpha_{k_{1}} \ldots \alpha_{k_{t-1}}} \sum_{x_{1} \in \mathcal{G}_{\alpha_{1}}} \ldots \sum_{x_{t-1} \in \mathcal{G}_{\alpha_{t-1}}} \chi \sum_{x_{t} \in \mathbb{F}_{p}^{*}} \chi\left(x_{1} \ldots x_{t}\right) \mathbf{e}_{p}\left(\Psi\left(x_{1} \ldots x_{t}\right)\right) \\
&=\frac{1}{\alpha_{k_{1}} \ldots \alpha_{k_{t-1}}} \sum_{x_{1} \in \mathcal{G}_{\alpha_{1}}} \ldots \sum_{x_{t-1} \in \mathcal{G}_{\alpha_{t-1}}} \sum_{x_{n} \in \mathbb{F}_{p}^{*}} \chi\left(x_{1} \ldots x_{t}\right) \\
& \mathbf{e}_{p}\left(a_{1}\left(x_{2} \ldots x_{t}\right)^{k_{1}}\right) \ldots \mathbf{e}_{p}\left(a_{t-1}\left(x_{1} \ldots x_{t-2} x_{t}\right)^{k_{t-1}}\right) \mathbf{e}_{p}\left(a_{t}\left(x_{1} \ldots x_{t}\right)^{k_{t}}\right) \\
&=\frac{1}{\alpha_{k_{1}} \ldots \alpha_{k_{t-1}}} \sum_{x_{1} \in \mathcal{G}_{\alpha_{1}}} \ldots \sum_{x_{t-1} \in \mathcal{G}_{\alpha_{t-1}}} \\
& \sum_{x_{t} \in \mathbb{F}_{p}^{*}} \omega_{1}(\mathbf{x}) \ldots \omega_{t}(\mathbf{x}) \mathbf{e}_{p}\left(a_{t}\left(x_{1} \ldots x_{t}\right)^{k_{t}}\right) .
\end{aligned}
$$

Now the image $\mathcal{X}_{t}=\left\{x_{t}^{k_{t}}: x_{t} \in \mathbb{F}_{p}^{*}\right\}$ of non-zero $k_{t}$ th powers contains $(p-1) / \alpha_{k_{t}}$ elements, each appearing with multiplicity $\alpha_{k_{t}}$. Similarly, the images $\mathcal{X}_{i}=\left\{x_{i}^{k_{t}}: x_{i} \in \mathcal{G}_{\alpha_{k_{i}}}\right\}$ contain $\alpha_{k_{i}} / \operatorname{gcd}\left(\alpha_{k_{i}}, \alpha_{k_{t}}\right)$ elements, each appearing with multiplicity $\operatorname{gcd}\left(\alpha_{k_{i}}, \alpha_{k_{t}}\right)$, for $i=1, \ldots, t-1$. Hence, we apply Lemma 3.4 to obtain

$$
\begin{aligned}
& T_{\chi}(\Psi) \ll_{t} \frac{\alpha_{k_{t}}}{\beta_{k_{1}} \ldots \beta_{k_{t-1}}} \cdot\left(p^{\frac{1}{2^{t}}} \beta_{k_{t-1}} A_{t}\left(\frac{p-1}{\alpha_{k_{t}}}\right) \prod_{i=1}^{t-2} B_{t}\left(\beta_{k_{i}}\right)\right) \\
& +\frac{\alpha_{k_{t}}}{\beta_{k_{1}} \ldots \beta_{k_{t-1}}} \cdot \frac{p-1}{\alpha_{k_{t}}} \beta_{k_{1}} \ldots \beta_{k_{t-1}}\left(\left(\frac{\alpha_{k_{t}}}{p-1}\right)^{\frac{1}{2}}+\beta_{k_{1}}^{\frac{-1}{2^{2}}}+\cdots+\beta_{k_{t-1}}^{\frac{-1}{2^{t}}}\right) .
\end{aligned}
$$

By simplifying we reach the required result. 


\section{Weyl Sums Over Generalized Arithmetic Progressions}

4.1. Preliminaries. We will require an estimate for the $\ell_{1}$ norm of the Fourier transform of proper generalized arithmetic progressions which is due to Shao [24].

Lemma 4.1. Let $\mathcal{A} \subseteq \mathbb{F}_{p}$ be a proper generalized arithmetic progression of rank $r$ and let $\widehat{\mathcal{A}}(z)$ denote the Fourier transform of $\mathcal{A}$. Then we have

$$
\sum_{z=1}^{p}|\widehat{\mathcal{A}}(z)| \ll_{r} p(\log p)^{r} .
$$

The following is due to Bourgain [3, Theorem A].

Lemma 4.2. Let $0<\delta<1 / 4$ and $r \geqslant 2$. There exists some $\delta^{\prime}$ such that if $p$ is a sufficiently large prime and $A_{1}, \ldots, A_{r} \subseteq \mathbb{F}_{p}$ satisfy

$$
\begin{aligned}
& \left|A_{i}\right|>p^{\delta} \\
& \prod_{i=1}^{r}\left|A_{i}\right|>p^{1+\delta},
\end{aligned}
$$

then

$$
\left|\sum_{x_{1} \in A_{1}, \ldots x_{r} \in A_{r}} e_{p}\left(x_{1} \ldots x_{r}\right)\right| \ll\left|A_{1}\right| \ldots\left|A_{r}\right| p^{-\delta^{\prime}} .
$$

4.2. Proof of Theorem 1.4. Considering the sum

$$
S=\sum_{a \in \mathcal{A}} e_{p}(F(a))
$$

we note that for any $a_{2}, \ldots, a_{d} \in \mathcal{A}$

$$
\begin{aligned}
S & =\frac{1}{p} \sum_{z=1}^{p}\left(\sum_{a \in \mathcal{A}} e_{p}(-z a)\right) \\
& \times \sum_{a_{1} \in d \mathcal{A}} e_{p}\left(F\left(a_{1}-a_{2}-\cdots-a_{d}\right)+z\left(a_{1}-a_{2}-\cdots-a_{d}\right)\right),
\end{aligned}
$$

where $d \mathcal{A}$ denotes the $d$-fold sumset

$$
d \mathcal{A}=\left\{a_{1}+\cdots+a_{d}: a_{i} \in \mathcal{A}\right\},
$$

so that

$$
|d \mathcal{A}| \ll|\mathcal{A}|
$$


Averaging (4.1) over $a_{2}, \ldots, a_{d} \in \mathcal{A}$ and using Lemma 4.1 gives

$$
S \ll \frac{1}{p} \sum_{z=1}^{p}|\widehat{\mathcal{A}}(z)| \frac{|T(z)|}{|\mathcal{A}|^{d-1}} \ll_{r} \frac{p^{o(1)}\left|T\left(z_{0}\right)\right|}{|\mathcal{A}|^{d-1}},
$$

for some $z_{0} \in \mathbb{F}_{p}$, where

$$
T(z)=\sum_{\substack{a_{1} \in d \mathcal{A} \\ a_{2}, \ldots, a_{d} \in \mathcal{A}}} e_{p}\left(F\left(a_{1}-a_{2}-\cdots-a_{d}\right)+z\left(a_{1}-a_{2}-\cdots-a_{d}\right)\right) .
$$

Since $F$ has degree $d$, we may write

$$
F(z)=\sum_{i=0}^{d} b_{i} z^{i}
$$

and hence

$$
\begin{aligned}
& F\left(a_{1}-a_{2}-\cdots-a_{d}\right)=\sum_{i=0}^{d} b_{i}\left(a_{1}-a_{2}-\cdots-a_{d}\right)^{i} \\
& \quad=F_{1}\left(a_{2}, \ldots, a_{d}\right)+\cdots+F_{d}\left(a_{1}, \ldots, a_{d-1}\right)+(-1)^{d-1} b_{d} a_{1} \ldots a_{d},
\end{aligned}
$$

for some sequence of polynomials $F_{1}, \ldots, F_{d}$ where $F_{i}$ is independent of the variable $a_{i}$. This implies that

$$
\begin{aligned}
& T\left(z_{0}\right)= \\
& \sum_{\substack{a_{1} \in d \mathcal{A} \\
a_{2}, \ldots, a_{d} \in \mathcal{A}}} \omega_{1}\left(a_{2}, \ldots, a_{d}\right) \ldots \omega_{d}\left(a_{1}, \ldots, a_{d-1}\right) e_{p}\left((-1)^{d-1} b_{d} a_{1} \ldots a_{d}\right),
\end{aligned}
$$

for some sequence of weights $\omega_{1}, \ldots, \omega_{d}$ with $\omega_{i}$ independent of $a_{i}$. By Lemma 2.1

$$
\begin{aligned}
\left|T\left(z_{0}\right)\right|^{2^{d-1}} & \ll_{d}|\mathcal{A}|^{d 2^{d-1}-1} \\
& +|\mathcal{A}|^{d 2^{d-1}-2 d+1} \sum_{\substack{a_{j}, a_{j}^{\prime} \in \mathcal{A} \\
j \geqslant 2}}\left|\sum_{a_{1} \in d \mathcal{A}} e_{p}\left(b_{d} a_{1}\left(a_{2}-a_{2}^{\prime}\right) \ldots\left(a_{d}-a_{d}^{\prime}\right)\right)\right|,
\end{aligned}
$$

and by the Cauchy-Schwarz inequality

$$
\begin{aligned}
& \left|T\left(z_{0}\right)\right|^{2^{d}} \ll_{d}|\mathcal{A}|^{d 2^{d}-2} \\
& +|\mathcal{A}|^{d 2^{d}-2 d}\left|\sum_{\substack{a_{j}, a_{j}^{\prime} \in \mathcal{A} \\
j \geqslant 2}} \sum_{\substack{a_{1}, a_{1}^{\prime} \in d \mathcal{A} \\
j \geqslant}} e_{p}\left(b_{d}\left(a_{1}-a_{1}^{\prime}\right)\left(a_{2}-a_{2}^{\prime}\right) \ldots\left(a_{d}-a_{d}^{\prime}\right)\right)\right| .
\end{aligned}
$$


This implies that

$$
\left|T\left(z_{0}\right)\right|^{2 d} \ll_{d}|\mathcal{A}|^{d 2^{d}-2}+|\mathcal{A}|^{d 2^{d}-d}\left|\sum_{\substack{a_{j} \in \mathcal{A}+c_{j} \\ j \geqslant 2}} \sum_{a_{1} \in d \mathcal{A}+c_{1}} e_{p}\left(b_{d} a_{1} \ldots a_{d}\right)\right|,
$$

for some $c_{1}, \ldots, c_{d} \in \mathbb{F}_{p}$. We note that the assumption (1.7) implies that the conditions of Lemma 4.2 are satisfied and hence

$$
\left|T\left(z_{0}\right)\right|^{2 d} \ll_{d}|\mathcal{A}|^{d 2^{d}} p^{-\delta^{\prime}}
$$

for some $\delta^{\prime}>0$ depending on $\varepsilon$ and the result follows from (4.4).

\section{REFERENCES}

[1] J. Bourgain, Mordell's exponential sum estimate revisited, J. Amer. Math. Soc. 18 (2005), no. 2, 477-499.

$[2] \_$, Sum-product theorems and exponential sum bounds in residue classes for general modulus, C. R. Math. Acad. Sci. Paris 344 (2007), no. 6, 349-352.

[3] _ Multilinear exponential sums in prime fields under optimal entropy condition on the sources, Geom. Funct. Anal. 18 (2009), no. 5, 1477-1502.

[4] , Estimates on polynomial exponential sums, Israel J. Math. 176 (2010), 221-240.

[5] - On exponential sums in finite fields, An irregular mind, Bolyai Soc. Math. Stud., 21, 2010, 219-242.

[6] J. Bourgain and M. Z. Garaev, On a variant of sum-product estimates and explicit exponential sum bounds in prime fields, Math. Proc. Cambridge Philos. Soc. 146 (2009), no. 1, 1-21.

[7] J. Bourgain, A. A. Glibichuck and S. V. Konyagin, Estimates for the number of sums and products and for exponential sums in fields of prime order, J. Lond. Math. Soc. 2, (2006), 380-398.

[8] J. Bourgain, S. Konyagin, Estimates for the number of sums and products and for exponential sums over subgroups in fields of prime order, C. R. Acad. Sci. Paris 337 (2003), 75-80.

[9] T. Cochrane, J. Coffelt, and C. Pinner, A further refinement of Mordell's bound on exponential sums, Acta Arith. 116 (2005), no. 1, 35-41.

[10] _ A system of simultaneous congruences arising from trinomial exponential sums, J. Théor. Nombres Bordeaux 18 (2006), no. 1, 59-72.

[11] T. Cochrane and C. Pinner, An improved Mordell type bound for exponential sums, Proc. Amer. Math. Soc. 133 (2005), no. 2, 313-320.

[12] — Using Stepanov's method for exponential sums involving rational functions, J. Number Theory 116 (2006), no. 2, 270-292.

[13] bridge Philos. Soc. 149 (2010), no. 2, 217-227.

[14] _ Explicit bounds on monomial and binomial exponential sums, Q. J. Math. 62 (2011), no. 2, 323-349. 
[15] M. Z. Garaev, Sums and products of sets and estimates for rational trigonometric sums in fields of prime order, Uspekhi Mat. Nauk 65 (2010), no. 4(394), 5-66.

[16] D.R. Heath-Brown and S.V. Konyagin, New bounds for Gauss sums derived from $k$-th powers and for Heilbronns exponential sum, Q. J. Math. 51 (2000), no. 2, 221-235.

[17] S. Macourt, Bounds on exponential sums with quadrinomials, J. Number Theory 193 (2018), 118-127.

[18] _ Incidence results and bounds of trilinear and quadrilinear exponential sums, SIAM J. Discrete Math. 32 (2018), no. 2, 815-825.

[19] S. Macourt, I. D. Shkredov, and I. E. Shparlinski, Multiplicative energy of shifted subgroups and bounds on exponential sums with trinomials in finite fields, Canad. J. Math. 70 (2018), no. 6, 1319-1338.

[20] L.J. Mordell, On a sum analogous to a Gauss sum, Q. J. Math. 3 (1932), 161-162.

[21] B. Murphy, G. Petridis, O. Roche-Newton, M. Rudnev, and I. D. Shkredov, New results on sum-product type growth over fields, arXiv:1702.01003

$[22]$ G. Petridis and I. E. Shparlinski, Bounds on trilinear and quadrilinear exponential sums, J. Anal. Math. (to appear).

[23] M. Rudnev, On the number of incidences between planes and points in three dimensions, Combinatorica, 2017, doi:10.1007/s00493-016-3329-6.

[24] X. Shao, On character sums and exponential sums over generalized arithmetic progressions, Bull. Lond. Math. Soc., 45, (3), (2013), 541-550.

[25] I. D. Shkredov, On exponential sums over multiplicative subgroups of medium size, Finite Fields Appl., 30 (2014), 72-87.

[26] — On asymptotic formulae in some sum-product questions, arXiv:1802.09066

[27] I. E Shparlinski, On bounds of Gaussian sums, Mat. Zametki 50 (1991), $122-130$.

[28] André Weil, Basic number theory, Classics in Mathematics, Springer-Verlag, Berlin, 1995, Reprint of the second (1973) edition.

Department of Pure Mathematics, University of New South Wales, Sydney, NSW 2052, Australia

E-mail address: b.kerr@adfa.edu.au

Department of Pure Mathematics, University of New South Wales, Sydney, NSW 2052, Australia

E-mail address: s.macourt@unsw.edu.au 